\title{
AUTOMOTIVE WIRING HARNESS DEVELOPMENT WITH THE USE OF „FOLLOW THE SUN" - TEAMS LOCATED IN DIFFERENT TIME ZONES
}

\author{
Tomasz Macura, Anna Timofiejczuk
}

Silesian University of Technology, Faculty of Mechanical Engineering, Department of Fundamentals of Machinery Design, Konarskiego 18A, 44-100 Gliwice

Corresponding author: Tomasz Macura, Tomasz.Macura@polsl.pl

\begin{abstract}
Demands of automotive industry is especially focused on new solutions. Nowadays, these needs are stronger than have ever been. Manufacturers are constantly looking for new ways to release their products in the shortest possible time. The research described in the paper concerns work organization and project management in automotive industry. It is a part of $\mathrm{PhD}$ project, dedicated to the implementation of remote team collaboration, and is focused on wiring harness development.

The first part of the paper is devoted to the review of different forms of work organization with special attention paid to Follow The Sun approach. In the second part of the paper, the characteristic of the wiring harness production was described. Especially, the methodology and design processes. The third part of the article presents a proposition of transforming the currently existing development process with the use of remote teamwork solutions. The article concludes with a description of the implementation of the test projects. Selected indicators were introduced to determine the profitability of this implementation.
\end{abstract}

Key words: Follow the Sun, time zones, organization of work, process management, remote teams, wiring harness, development, automotive

\section{INTRODUCTION}

Nowadays, a physical distance between two people or even whole project teams is no more a barrier in performing difficult and complex projects. The existence of broadband Internet has brought to the table the great solutions for shortening the physical distance and gave the opportunity to flawless cooperation. The implementation of such approaches came out even more important in pandemic time. Presently, automotive companies are constantly searching for new optimal solutions and ideas to achieve and improve the leader position in the business. Constant optimization of ongoing processes and internal procedures is the key to achieve that goal. One of the main trends in automotive industry is investing and setting new locations in countries which are currently in a phase of intensive development. Such countries can offer significant amount of engineers at a very reasonable cost. To take advantage of such diverse human resources numerous approaches could be applied. One of them is a concept of working in remote teams located in different time zones. There are series of engineering activities and tasks that could be performed in form of distance cooperation. Examples are elaborating software and conducting designing projects. The approach taken into account in the presented research is called Follow The Sun (FTS). Its implementation leads to several new challenges the companies must face up to establish good cooperation between different locations spread all over the world. Considering the designing process as well as main aspects of the FTS approach one can distinguish common features and routine tasks. They are described with the use of numerous data and information. To simplify the FTS-based management as well as the design process, some parts of the approach can be generalized using context related to similar or the same circumstances.

\section{OVERVIEW - TYPES OF TEAMS COOPERATION WORKFLOW}

According to the definitions, a team is a group of people performing collaborative work requiring collective efforts and benefiting from the created synergy. Not every group of people working together can be called a team. Required features that define the group as the team are: to have a common goal, a common system of values and norms, collective responsibility and mutual support (Ćwik, 2013). In the literature there are four main types of teams described:

Functional work team - this is the most common type of team in companies, where all members work in the same department and report to the same manager.

Cross-functional team - a team formed by employees 
from different departments of a given company, usually at the same level in the corporate hierarchy. Such teams are most often established during work on processes affecting the whole company.

Self-managing team - a team established by people working together in the same organization. The team has a clear objective but has no leader to coordinate the work. In this type of team, communication and feedback are extremely crucial.

Virtual team - this is a very specific team since it brings together specialists from the same organization working on a daily basis in different locations. Cooperation in this type of team takes place only and exclusively with the use of remote teamwork tools. This type of solution allows to build a strong team consisting of the best professionals in the organization.

\subsection{Time zone-based team management}

The first example of the time zones-based cooperation concept was implemented a couple years after launching Internet as a commercial tool of communication. Examples of FTS implementations were described in paragraph 3. The first company to try this approach was IBM. Under the lead of CEO Louis V. Gerstner Jr., the first intercontinental software development project group was launched. It consisted of 5 teams, one in U.S. and four placed in Eastern Hemisphere. The U.S team was leading the whole operating process and was responsible for setting the tasks, then at the end of the day sending them to other four teams for further development coding. Every next day the U.S. based team was responsible for reviewing and checking (Carmel, Espinosa, 2011).

Next years, the approach was implemented in other companies and developed as two kinds Follow The Sun or Round the Clock. They are distinct from each other, but unfortunately these terms are used very often interchangeably. It is a very common mistake since both approaches are being used for completely different purposes. The round the clock method means a cooperation focusing on very similar tasks, in most cases easy to solve and generic. A good example of this approach is 24 hours, 7 days a week IT Support. Tasks are mostly similar and not complex. Usually there is no need for continuing to perform the issue by the second team. Raised issues or by IT technical jargon called "Tickets" are being solved during one working day, by a particular team. In contrast, Follow The Sun is dedicated for far more complicated tasks. The fundamental feature of FTS is bringing a complex solution to the market in a very short time, faster than using conventional development approach. The FTS development process is being divided into separate tasks which can be performed by individual teams located in different time zones. Providing the opportunity to accelerate the development process by three times. To assure proper handover in FTS it is crucial to have at least one hour overlap between shifts of cooperating teams. Therefore, FTS cannot be implemented in any group of locations. They must be chosen carefully, so the time zones in which they are working are correctly joining together. The best approach is to have 3 locations, the first placed in America, the second in Europe and the last one in Asia. The split of hours China (GMT +8), Poland (GMT+2) and Mexico (GMT-5) is presented in Figure 1.

\begin{tabular}{|c|c|c|c|c|c|c|c|c|c|c|c|c|}
\hline & $22: 00$ & $00: 00$ & 02:00 & \begin{tabular}{|l|}
$04: 00$ \\
\end{tabular} & 06:00 & \begin{tabular}{|l|}
$08: 00$ \\
\end{tabular} & 10:00 & \begin{tabular}{|l|}
$12: 00$ \\
\end{tabular} & \begin{tabular}{|l|l|}
$14: 00$ \\
\end{tabular} & 16:00 & \begin{tabular}{|l|}
$18: 00$ \\
\end{tabular} & \begin{tabular}{|l|l|}
$20: 00$ \\
\end{tabular} \\
\hline China & & & & & & & & & & & & \\
\hline Poland & & & & & & & & & & & & \\
\hline Mexico & & & & & & & & & & & & \\
\hline
\end{tabular}

Fig. 1. Project teams time zones

The content and structure of the handover is directly related to the type of tasks being performed and the stage at which the project is currently in progress. It is crucial that the team coordinator performs his or her tasks proactively to define the form of the handover so that each successive team taking over the results of the previous one would spend as little time as possible to find out what phase the tasks are at. Due to the cultural differences between the teams, communication between them during the handover may be difficult. This can be observed especially in countries where the hierarchical structure of the teams is well grounded and there is a psychological distance between team members (Bieniasz, 2018). Therefore, it is good practice to use a formalized version of the handover, where both teams use a template prepared by the project coordinator during the meeting. The template contains a series of questions, moderating the sequence of the meeting, implying a predefined meeting structure. The predetermined course of the meeting allows the teams to prepare all necessary information in advance. The result of such activity makes it possible that handover time is reduced to minimum.

\subsection{Managing remote teams}

To establish a definition of remote teams, it is essential also to define the classic term for a team. Such definition together with selected kinds of teams were presented at the beginning of this paragraph. The size 
of the team is not limited and is closely related to the type of tasks performed by employees. However, it is well established practice in management literature not to exceed 12 people in one team coordinated by one manager (Katzenbach, Smith, 1993). A good team consists of members whose skills complement each other and together they create the synergy needed to achieve the desired goal. In some companies there are also initial tests related to personal and psychological features of members to collect the team of desired characteristics.

The difference between regular and remote teams is the physical location of team members. In case of the regular team, it is usually one room or office, while in case of remote teams it can be another city, country or even continent. As a result, remote teams characterize with advantages, but also challenges from the point of view of coordination. The advantages of dispersed teams are that they remove the restriction on creating teams with people from only one office. This allows gathering teams made up of the best specialists available now in the industry and flexible change in the occurrence of new tasks requiring additional expertise beyond the original competences of the team. Moreover, the possibility of creating a team formed from the best specialists allows a very fast exchange of knowledge and experience, significantly increasing creativity and innovation level in achieving the desired goal. However, remote teams raise huge challenges in terms of coordination. The lack of direct interpersonal contacts that take place in a regular team every day in the office leads to many mistakes in cooperation and communication, which is in such case based only on electronic medium. The lack of daily direct contacts causes also significant divisions within teams and creating smaller subgroups. It is therefore important for the coordination of this type of team to clearly define 3 basic criteria in team functioning: goal, division of tasks and communication (Kossler, 2004). Each member of the team must understand the purpose of the implemented tasks and their background in the global perspective of the company. The responsibilities of each team member should be clearly defined and transparent for the rest of the team members. Moreover, the time frame for the implementation of these tasks must be established and respected from the very beginning. The last pillar of proper functioning of the team is communication. The responsibility of the person coordinating the team is to plan an effective way of communication and its moderation already during the execution of a given challenge. This role should not be underestimated by managers because, as the examples of attempts to implement remote teams showed, the main reason for not meeting the deadlines for tasks or occurring problems was ineffective communication. A good practice applied when setting up remote teams is the organization of an integration meeting in a selected company office. This allows us to establish relations between team members and to build basic trust.

\subsection{Organization of remote working}

The key to organizing the cooperation between remote teams is to make parallel communication available. A great solution is to use different dedicated tools and platforms. An example is the most popular now Microsoft's Teams platform, which is a part of Office 365 package. With the use of the platform, it is not only possible to hold conversations and video-conferences but also to exchange files and work on files in parallel. The platform allows for daily project coordinating, analyzing individual tasks as well as making team decisions based on shared data storage and discussions. The research described in the paper is focused on teamwork related to design tasks. In such case the next key aspect is the CAD data sharing point. In the form of the classic cooperation, it was a file server provided and managed by the company. Currently, the latest trend is to move CAD data to the cloud. This solution brings several improvements but also some dangerous aspects. All design data is available from anywhere in the world at the same time, only the Internet service of good quality is required. The cloud service should be responsible for protecting data in case of damage of the computer or disk. The only disadvantage of this type of solution may be the potential ability to access project data by individuals who are not entitled to use it. Since that, the aspects of cybersecurity are required to be also considered as next crucial problem.

\section{EXAMPLES OF FOLLOW THE SUN IMPLEMENTATION}

The first well documented implementation of FTS started in 1990. IBM set up four equal sized low-cost offshore locations: Bangalore, India, Belarus, Latvia. The coordination of the project was placed in Seattle (Carmel et al., 2010). Each team consisted of thirtyone professionals with 5 leading core specialists. This structure was named Phalanx and was especially set up as the standard IBM low-cost location structure. Therefore, it was clear and easy to communicate across all locations and delegate specific tasks. The second purpose of such structure was establishing new locations by using 5 leading core experts.

IBM established special nomenclature for divided tasks, they were named JavaBean's. Each JavaBean could be developed separately and rapidly. Low-cost offshore locations were responsible for developing those beans, then during the day in U.S. there were checked and reviewed by Seattle location. In case the review of one bean took more than one day, the second bean was being developed parallelly. Each location received about 6 beans to develop. At the top of operating system 
there were more than 20 beans waiting for evaluation. Unfortunately, presented approach came out wrong form of teamwork for IBM. Daily handovers turn out to happened to often, managers have decided to make handovers every second or third day as standard rhythm. After couple of months the central hub Seattle turns out to be main blockage because of slow beans review respond. That led to hard escalation from the side of managers. In result they decided to give up on using globally distributed approach to this project and returned to classic management methods parallel or phase based. The project was so much behind that low-cost location decided to work during night shifts to maintain constant contact with Seattle. Eventually, the difference in time zones turned out to be recognized as the main problem instead of being expected advantage.

\section{WIRE HARNESS DESIGN PROCESS}

The implementation of the FTS method, which results were presented at the end of this article, was implemented in the area of electrical harness design for the automotive industry. In the following section the product of electrical harness is presented in order to better understand the specifics of this field and especially, the design process.

The vehicle's electrical wiring harness can be compared to the human nervous system. The main task of the wire harness is to supply power and ensure communication between all devices in a car. The wiring harness is also a highly customized product because its final form is directly determined by all the options the customer has configured in the car showroom.

The elements of the standard wiring harness used in the interior of a passenger car are electric cables with a cross-sectional area of $0.35 \mathrm{~mm}^{2}$ to $6 \mathrm{~mm}^{2}$, most often occurring in 10 basic colors: white, yellow, red, violet, blue, green, grey, brown and black. Groups of cables are terminated with plugs, where each single cable is terminated with a metal element called the contact, usually made from zinc. There are also contacts coated with noble metals, silver, or gold. For example, connection of airbags are coated with gold. Such elements are required to be highly reliable and fast in case of an accident. The electrical harnesses also include elements that protect the harness against the negative effects of the external environment such as vibration, temperature, or humidity. These are usually insulation tapes, rubber elements and protective sleeves.

The degree of sophistication and complexity of the wiring harness is directly related to the amount of equipment used in a particular car model. With the development of cars over the years, the number of devices the car is equipped with has significantly increased, and therefore also the number of wires included in the wiring harness needed to connect this equipment. During the design of the wiring harness, each additional electrical system is called a module. For example, the electric cables needed to operate the front seat heating function is called module $\mathrm{X}$, while the cables needed to operate the electric windscreens is called module Y. A perfect example of illustration of this increasing trend in the number of modules in the wiring harness over the years is Volkswagen Golf. In case of Golf 3 generation from 1993 had 48 modules of the wire harness, the Golf 5 generation from 2003 had already 359 modules, while the Golf 7 generation from 2013 had a complexity level of 410 modules. It is obvious that not all modules are built into a given harness, this is already decided by a customer during the configuration of equipment of the car at the order stage. The average number of modules built into the $7^{\text {th }}$ generation of Golf is 236 , which means that the total length of all electric wires used in the bundle is equal to $1580 \mathrm{~m}$ (Meyer, 2013).

The design process of the electrical harness, according to which all activities are carried out, is defined by the OEM customer. The Volkswagen group uses the VOBES process - Volkswagen Bordnetz Entwicklungs System. The diagram of this operating process is shown in Figure 2. It is applied in the following car brands: Volkswagen, Audi, Seat, Skoda, Bentley, Bugatti, Lamborghini, Porsche, and Man (Kyriazis, 2006).

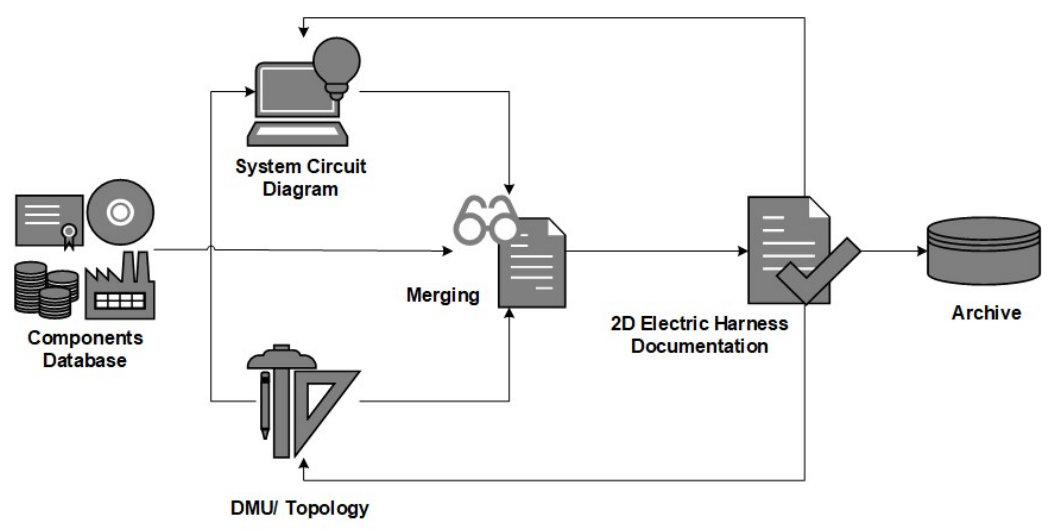

Fig. 2. Schematic Diagram of VOBES Process 
Depending on the type of a project, wiring harness development can take from one to three years. The fastest projects are realizations of wiring harnesses associated with minor body modifications called "face-lifts" of a certain car model. In this case, the entire project is based on a manufactured wiring harness for the current car model, and the customer provides new 3D models of these areas that change with the introduction of the face-lift model to the market. On the other hand, the most demanding and time-consuming wiring harness projects are related to completely new car models just joining the manufacturer's portfolio or renewed models after a long period of discontinuation of a given car. In these examples, the design process starts completely from scratch, and it is not possible to take advantages of previous drafts.

According to the presented diagram, the harness design process starts with the customer providing input data. Then, parallel works are carried out on the system of connections - "Electrology" and "Digital MockUp - DMU/Topology". Digital MockUp is a geometrical model of an electrical harness, whose external shape depends on the design of the car bodywork as well as placement of all the components fitted to the car. The development of the DMU is carried out in the 3D CAD modeling environment. Then, the results of work are combined in the form of $2 \mathrm{D}$ drawing, which is the basis for further steps of the electric harness manufacturing process.

\section{THE WIRING HARNESS MANUFACTURING PROCESS}

The production of electrical wiring harnesses is very demanding operation due to restrictive quality standards and a narrow margin of errors. It influences the safety of driving a car. The slightest failure of the wiring harness during the use of a car can result in a risk of fire threatening the lives of drivers as well as other road users.

The automotive industry has undergone significant changes in recent years in terms of automation of all production processes. Thus, it is surprising that the production process of the electric harnesses is still in most areas performed manually. This is caused by the very high complexity of this production as well as the requirement to flexible adjustment of the manufactured products to the orders received from the customer related to the type of equipment of a given car. Therefore, manual work in wiring harness production is still an optimal solution in terms of costs. The production process begins with cutting off all the necessary wires in required length according to the technical documentation provided by the design department. Then the wires are fitted with pins at the ends. The next step is to assemble individual modules consisting of a few to several wires. The final stage in the production of a wiring harness is the assembly of the entire product, which consists of the prepared single modules and other previously processed wires. It is performed on specially prepared assembly tables, this procedure is executed completely manually. The finally produced harness is put to the test for checking connection correctness, where each single cable is inspected. After passing the connection test, the wiring harnesses are packed in fabric bags and transported to the customer for final assembly.

\section{IMPLEMENTATION OF "FOLLOW THE SUN" APPROACH IN THE DESIGN OF WIRING HARNESS}

The main aim of the research described in the paper as well as included in the $\mathrm{PhD}$ thesis is to improve the design process of the wiring harness. Currently the process is performed separately by a few different teams located in different countries and there are numerous problems with communication. It is assumed that the implementation of FTS approach brings multiple beneficial effects on some processes and the on the company.

At present, in the standard operation of the development department, each project is carried out by one team in one location. This is a classic project approach that has been used and improved for over twenty years. It ensures high team stability and product quality. Introducing a new solution while maintaining the above standards would also entail a reduction in overall development time.

In order to illustrate the differences in the functioning of the project team after the introduction of the FTS solution, a diagram has been prepared, Figure 3. The upper part of the diagram shows the current workflow for the local team and the lower part the workflow for the virtual team. 


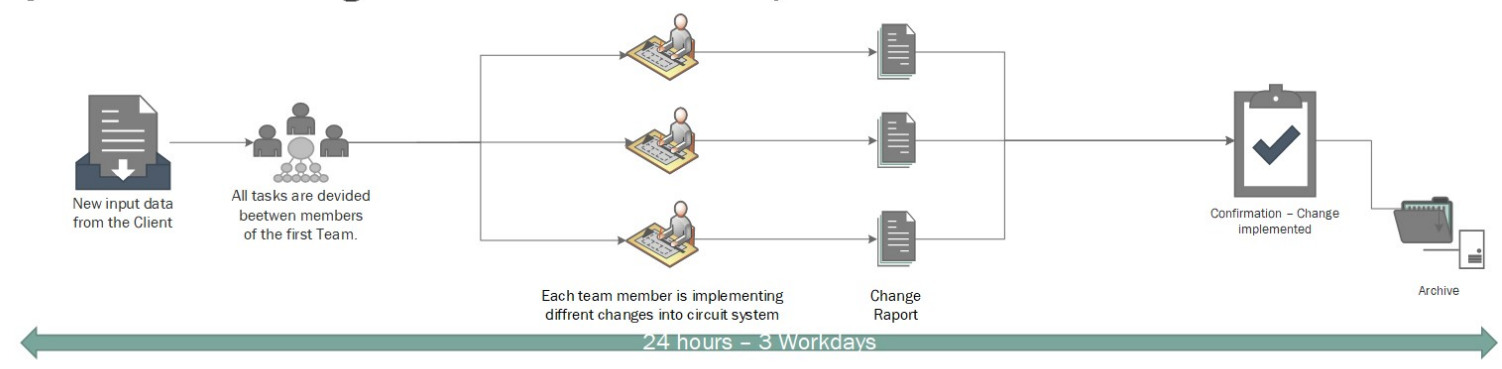

System Circuit - Multi location workflow setup

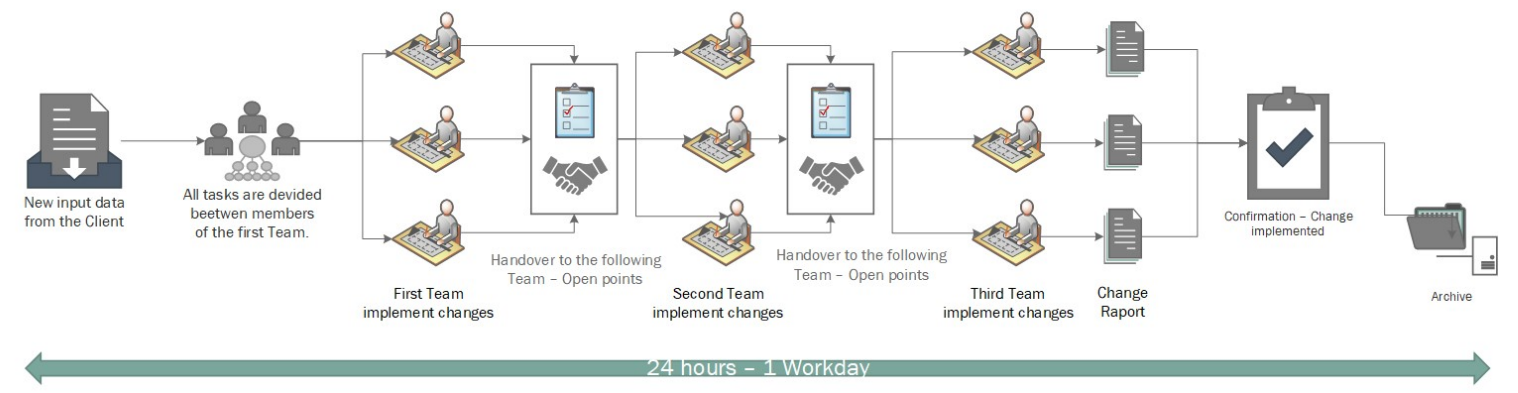

Fig. 3. Workflow diagram for single and multi-location setup

The classic workflow starts with the new input data received from the customer and divided between specific members of the team responsible for selected areas of the car interior. Then, within three working days, changes are implemented in the documentation of the electric wire harness.

The multi-location workflow starts with new input from the customer, similarly to the classic approach, divided into determined members of the first team working on a given project. Then, at the end of the first team's working day, the "Handover" takes place, so that currently implemented tasks are transferred to the second team, which is starts work in another location. During the handover all open issues and topics that need clarification are discussed. Then, at the end of the second team's work day, as in the previous case, the tasks are handed over to a third team, located in a different location. The total time for all documentation changes is one working day.

\section{EFFECTIVENESS ANALYSIS OF IMPLEMENTATION THE "FOLLOW THE SUN" APPROACH}

The main objective of the described research is to conduct a proof of concept in the implementation of FTS solution into the wiring harness design process. During the first phase of the implementation, a special test project carried out at three locations was performed. The completion of this phase has provided a number of valuable information in topic of the profitability of this type of solution in comparison to the currently used design methodology, but also to define a number of difficulties that occur in this type of endeavor (Carmel et al., 1999).

The following indicators are used to verify the beneficial impact on the product development process resulting from the implementation of the FTS solution. The indicators are divided into two groups. The first group consists of profitability indicators including DTC, DHC, DTC, TTC, ER, PS, which are quantity characteristics. The second group are quality indicators, which include: MS, TF. The indicator together with the method of their calculation are presented in Table 1.

Table 1. Indicators used to evaluate the effectiveness of the "Follow The Sun" method

\begin{tabular}{|c|c|c|}
\hline & Indicator & Calculation method \\
\hline |DTC| & Design Time Cost & $\begin{array}{l}\text { Amount of engineering hc } \\
\text { complete the project }\end{array}$ \\
\hline I DHC | & Design Hours Cost & Cost of engineer's design $h$ \\
\hline IDSCl & Design Support Cost & $\begin{array}{l}\text { Cost of support (software a } \\
\text { needed to complete the pro }\end{array}$ \\
\hline | TTC | & Time To Complete & $\begin{array}{l}\text { Amount of business days I } \\
\text { plete the project }\end{array}$ \\
\hline |ER | & Error Rate & $\begin{array}{l}\text { Amount of errors reported } \\
\text { tor }\end{array}$ \\
\hline | PS | & Process Stability & $\begin{array}{l}\text { Number of events requirin } \\
\text { the coordinator in informat }\end{array}$ \\
\hline
\end{tabular}

Special attention should be given to the Time to Complete (TTC) indicator. Its value can be defined as a partial value of the indicator widely used in the automotive industry - Time To Market (TTM). It is 
defined as a summarized time needed to introduce a new product on the market, from the very beginning of concept works to the production of a ready product (Bręk, 2018). The TTC indicator defines a fraction of the total development process of a given product, in which a clearly defined package of modifications to the product design is implemented.

\section{TEST IMPLEMENTATION OF "FOLLOW THE SUN" IN THE AREA OF ELECTRICAL HARNESS DESIGN}

In order to provide an initial test of the "Follow The Sun" method in the work organization of the team responsible for designing electrical harnesses, a test implementation was carried out on a smaller group of employees.

A team of 6 participants in the test was established. The team consisted of 2 managers and 4 design engineers. Due to available options and project phase, the trial was conducted on a team working in two countries: Poland and Mexico. The two design offices were 7 hours apart from each other from a time zone perspective.

The object of the test was to carry out development of a 3D model of electric harnesses for an electric car. The aim of the project was to adjust the shape of electric harnesses according to the modified shape of the chassis. In order to carry out a comparative test, 3 models with a similar degree of complexity and requiring a comparable amount of work by the engineer were selected from all the harnesses requiring modification.

The development of the first harness model was carried out in the classical design process by an engineer from Poland. The work on the second harness model was carried out in the same way by an engineer from Mexico. The work on the third harness model was carried out by a team of two engineers from Poland and Mexico using the "Follow The Sun" method. This distribution of tasks made it possible after the test to compare the effectiveness of the "Follow The Sun" method by following the indicators presented in the previous paragraph.

Engineers were informed prior to the start of the project about the test being conducted, but instructed not to change the standard tempo of the work so as not to affect the results. The task completion time was set at 13 working days. The results of the conducted test are presented in Table 2.

Table 2. Results from a test implementation

\begin{tabular}{lll}
\hline IDTCI & | TTC I & Project \\
\hline 80 Hours & 10 days & Harness Model 1 - Engineer \\
88 Hours & 11 days & Harness Model 2 - Engineer
\end{tabular}

In order to visualize the obtained results, they have been presented in the form of diagrams in the Figure 4.

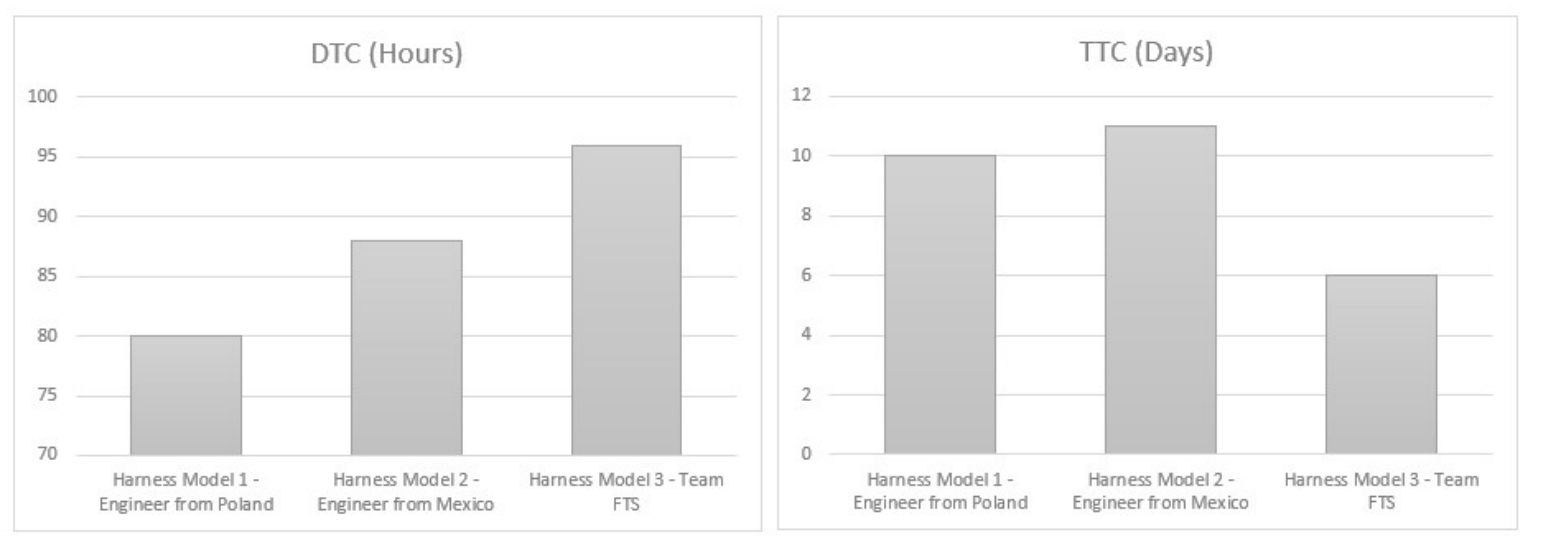

Fig. 4. Graphical presentation of the obtained DTC and TTC indicator results

Based on the carried out test, the following observations were made:

-The team working in the FTS formula needed 6 days to complete the task compared to 10 and 11 days for the classical wiring harness modelling approach. This resulted in a TTC - Time to Complete ratio 43\% lower than in the classic process. This is a surprisingly high result considering the first implementation of this method.

-For the DTC indicator, which is the sum of hours needed to complete the given task, an increase was observed for the FTS method. After averaging the number of hours needed to complete a comparable task by an engineers from Mexico and from Poland. The increase in the amount of design hours in the case of the FTS formula is 12 hours which represents $14.3 \%$ increment.

-Lack of handover, breaks the chain of contacts and requires the next engineer to work harder to find the changes made by the previous person.

-During the implementation of the FTS method, it is important to define the responsibilities of individual team members. One person should coordinate the 
work on the product in a given way and consider himself responsible for it.

-In the case of engineers with less experience, a certain hesitation or lack of confidence in communication is noticeable. This results in communication difficulties, which in the case of the FTS method have a significant influence on the collaboration.

-The inability to meet face-to-face with all participants prior to testing, due to the pandemic, resulted in communication difficulties between participants during the project.

-The different lead times for the Mexican and Polish engineers are due to the difference in experience of each engineer as well as to the slightly different design of each electrical harness.

-Due to the specification and dynamics of the electrical harness development, any testing and analysis of the FTS implementation is affected by external factors such as the experience of the individual engineers involved, the differing complexity of the electrical harness models, and random human error.

\section{CONCLUSIONS}

The approach of "Follow The Sun" presented in the paper can be successfully implemented in the area of wiring harness design. The potential benefits of this type of approach are very significant and can greatly strengthen the position of the company in one of the most competitive industries such as automotive.

The variety of tools introduced to enable collaboration between teams located in three different locations can also be used to full advantage when introducing remote working capabilities for each location.

However, the implementation of such a solution is very complex and requires a holistic approach. This is also confirmed by the scientific publication on the subject. There are not many descriptions of successful implementations of this type of solutions in the literature. Moreover, the literature focuses on implementations in the broad IT area. The noticeable influence of external conditions on the work of engineers such as differences in experience, mentality, motivation, communication and many others emphasizes the necessity of using context (Timofiejczuk, 2011) in the diagnosis of such complex structures of cooperation (Pollak et al., 2020).

The results of the conducted test provided a number of useful information for planning the implementation of this type of work system in other branches of the automotive industry product development, not only strictly the area of electrical harness development. Conducted tests are also a great foundation for the realization of further tests on a larger group of engineers as well as on a broader range of design office locations.

\section{REFERENCES}

1. Carmel E., Espinosa J. A., Dubinsky Y., (2010). "Follow the Sun" Workflow in Global Software Development. Journal of Management Information Systems archive, 27(1), 17-39.

2. Carmel E., Espinosa J. A., Dubinsky Y., (1999). Global software teams : collaborating across borders and time zones. London: Prentice Hall International.

3. Kroll J., Hess R. E., Audy N. L. J., Prikladnicki R., (2011). Researching into Follow-the-Sun Software Development: Challenges and Opportunities. Conference: $6^{\text {th }}$ IEEE International Conference on Global Software Engineering, ICGSE 2011, Helsinki, Finland, August 15-18, 2011.

4. Carmel E., Espinosa, J. A., (2011). I'm working while they're sleeping - Time zone separation challenges and solutions. United States of America: Nedder Stream Press. 5. Kossler M., (2004). Leading Dispersed Teams. Greensboro: Centre for creative leadership, 28.02.2004

6. Timofiejczuk A., (2011). Methodology of context based reasoning in technical diagnostics. Wydawnictwo Politechniki Śląskiej.

7. Meyer D., (2013). Das Golf 7 Bordnetz. Conference: Fachkongree Bordnetze im Automobil, April 2013.

8. Kyriazis J., (2006). Integration of the Electrical System Development Processes with KBL. Conference: ProSTEP iViP Symposium 2006.

9. Bręk Ł., (2018). TTM stands for Time To Market (Polish translation: TTM czyli Time To Market). Available from: https://bialko.eu/agile/ttm-time-tomarket, Accessed: 02/08/2021.

10. Bieniasz M., (2018). Distributed teams - what challenges do they present to the manager? (Polish translation: Rozproszone zespoty - jakie wyzwania stawiaja przed managerem?). Available from: https://kjarocka.pl/zarzadzanie-projektami/rozproszonezespoly, Accessed: 02/08/2021.

11. Ćwik K., (2013). Teamwork (Polish translation: Praca zespolowa). Available from: www.hs.dobrekadry.pl/docs/ Accessed: 02/08/2021.

12. China's economy to grow by $9 \%$ in 2021 . Available from: https://atalayar.com/en/content/ chinas-economygrow-9-2021, Accessed: 02/08/2021.

13. Katzenbach JR, Smith DK (1993) The wisdom of teams: Creating the high-performance organization. Harvard Business Review Press, Boston, Massachusetts. 14. Pollak, A., Hilarowicz, A., Walczak, M. and Gąsiorek, D. (2020). A Framework of Action for Implementation of Industry 4.0. an Empirically Based Research. Sustainability, 12(14), p.5789.

Received: August 16, 2021 / Accepted: December 15, 2021 / Paper available online: December 20, 2021 (C) International Journal of Modern Manufacturing Technologies 